\title{
EL TURISMO EN LOS PROCESOS DE DESARROLLO RURAL
}

\author{
José Luis Andrés Sarasa ${ }^{1}$ \\ Universidad de Murcia
}

\section{RESUMEN}

El objetivo de este trabajo se concreta en presentar las etapas que deben seguirse para alcanzar un desarrollo rural integrado. Para ello se propone reflexionar en torno a la nueva funcionalidad del medio rural, las disfuncionalidades que atenazan su desarrollo, las ventajas cualitativas en que apoyarse y los actores y estrategias que deben entrar en juego. Se concluye que posicionarse en la jerarquía territorial emergente no puede ser a cualquier precio y que no es tarea fácil, por ello es preciso movilizar todas las sinergias locales.

Palabras clave: Gobernanza, desarrollo, estrategias, patrimonio, disfuncionalidades, historia local, actores locales, rehabilitación, revitalización, gestión, internacionalización, atraso estructural.

\section{TOURISM IN RURAL DEVELOPMENT PROCESSES}

\begin{abstract}
The aim of this paper is to present the various steps required to achieve integrated rural development. This requires thinking about the new functionality of rural areas, the dysfunctions that affect their development, qualitative advantages and actors and strategies that must come into play. We conclude that position in the emerging spatial hierarchy can not be done at any price, and it is not easy, so it is necessary to mobilize all local synergies.

Key words: governance, development, strategies, heritage, dysfunctions, local history, local actors, rehabilitation, revitalization, management, internationalization, structural, backwardness.
\end{abstract}

Fecha de recepción: 27 de Marzo de 2014. Fecha de aceptación: 25 de Noviembre de 2014.

1 Departamento de Geografía. Facultad de Letras. Universidad de Murcia. Campus de la Merced. 30001 Murcia (España) e-mail: jlandres@um.es 


\section{INTRODUCCIÓN}

El proceso de globalización que impregna la vida económica, social, cultural y política fuerza a todos los ámbitos territoriales, sean cuales sean sus características y tamaño, a posicionarse para desempeñar una nueva función: la internacionalización. Los Corredores transeuropeos son un buen ejemplo y una oportunidad Todo espacio rural -Comarca del Jiloca, Comunidad de Teruel, etc.- que quiera existir en la nueva «jerarquía territorial» debe convertirse de alguna manera en nodo global, capaz de hacer frente a los desafíos que plantea ajustarse a cambios fundamentales en ordenación del territorio y desarrollo regional, comarcal y local.

El nuevo modelo de desarrollo debe apostar por encontrar un proyecto que no sólo persiga los objetivos de la Estrategia de Lisboa en su relanzamiento, sino que sea capaz de situar al territorio en condiciones de competir en la jerarquía emergente, donde lo que no es agradable, consumible, previsible y deseado no tiene sitio. Esta pretensión exige, en primer lugar, tener un mínimo de ambición internacional porque parodiando a Amendola, ${ }^{2}$ «hoy, el nuevo medio rural también puede y debe vivir de las transacciones y de los intercambios simbólicos, más que de la producción de bienes. Urge orientarse hacia el exterior y a las interconexiones, encontrar la gran oportunidad en la imagen y en la capacidad de atracción». Este ambicioso objetivo, requiere implicar a un amplio conjunto de actores (responsables políticos, empresarios, personalidades científicas, económicas y culturales, universidades, asociaciones, etc.), estrategias y recursos patrimoniales y humanos, por todo ello es preciso poner en práctica una nueva gobernanza del medio rural, entendida como resultado del diálogo entre todos los actores comprometidos con el desarrollo rural. Hoy por hoy constituye un nuevo paradigma para la gestión territorial al que debe aferrarse un medio rural necesitado de una estrategia de ordenación para hacer frente a los retos que tiene ante sí..

El objetivo de este trabajo se propone presentar las etapas que deben seguirse para poner en práctica una nueva gobernanza que conduzca a un desarrollo local que, valorizando el patrimonio como capital insustituible a la hora de confeccionar una nueva imagen, permita al medio rural posicionarse en interconexiones exteriores. Abordar tan complejo objetivo exige, en primer lugar, reflexionar en torno a los objetivos, instrumentos, premisas y cimientos que sustentan la nueva funcionalidad. En la segunda etapa se propone un detenido recorrido por el territorio para descubrir las disfuncionalidades estructurales surgidas como consecuencia de las complejas interrelaciones causa/efecto del reciente desarrollo. La siguiente etapa se concreta en aprovechar la ventaja cualitativa de la historia local, definida por la valoración del patrimonio material e inmaterial como instrumentos capaces de responder a las exigencias de la nueva función. Por último, es preciso identificar los diferentes actores con sus respectivas estrategias que serán capaces de movilizar todas las sinergias locales, comarcales y regionales, cuyos objetivos generales se concreten en apostar por un territorio sostenible, promoviendo la rehabilitación y revitalización de los saberes locales a través del patrimonio material e inmaterial.

2 AMENDOLA, G. (2000): La ciudad postmoderna. Ediciones Celeste. Madrid. Pp. 180. 


\section{FUNDAMENTOS DEL NUEVO PROYECTO DE ESPACIO RURAL}

La literatura especializada pone de manifiesto que toda política elaborada con el propósito de posibilitar a cualquier territorio vivir y prosperar en procesos de globalización tiene que integrarse en una dimensión internacional. Al igual que ha ocurrido con el mundo de las empresas, pero también a nivel individual de las personas, que si pretenden alcanzar el éxito deben planificar su futuro en una óptica cada vez más internacional, el medio rural no puede quedarse al margen de este fenómeno y debe admitir que su desarrollo y capacidad competitiva gozará de mayores expectativas si se gesta integrada en una marco que supere los meros límites locales, comarcales o regionales.

El Libro Verde del Medio Ambiente Urbano en el ámbito de la gestión urbana afirma que «las dinámicas globales de cambio han ido modificando de forma sustancial el escenario en el que las relaciones entre esferas de gobierno y actores públicos y privados desarrollan sus estrategias. La globalización económica, acompañada por la emergencia de una cultura global, ha alterado profundamente la realidad social, económica y política de los estados, las regiones y las ciudades». ${ }^{3}$

La internacionalización se convierte, por esta razón, en prioridad para todo proyecto del espacio rural que se proponga hacer frente a cuantos desafíos plantea ajustarse al cambio estructural de la economía y con ella de la vida cotidiana. Existen abundantes evidencias de las ventajas que obtienen aquellos pueblos y pequeñas ciudades que apuestan con decisión por este nuevo modelo de desarrollo, respecto de quienes permanecen en una incomprensible pasividad para lamentarse de sufrir los efectos negativos de la ciudad global, no son capaces de beneficiarse de las oportunidades que ofrece el nuevo modelo basado en la internacionalización.

En este sentido, el citado Libro Verde matiza: «El desarrollo de la UE supone una experiencia de gobernanza transnacional: en tanto que organización política emergente no se basa en los principios tradicionales de soberanía y exclusividad territorial, sino que se define como un ejercicio difuso y compartido de la autoridad. Establece un sistema de gobierno multinivel y relacional: la negociación sustituye a la autoridad como recurso fundamental para dotarse de la capacidad de gobernar. Y la red va sustituyendo al territorio físico como referencia espacial sobre la que aplicar las capacidades de gobierno».

Arriesgar por la puesta en práctica de esta estrategia, apostar por un nuevo proyecto de espaio rural exige reflexionar sobre cuatro factores que a nuestro juicio sustentan el modelo de desarrollo que se propone: objetivos, instrumentos, condición básica y cimientos.

En primer lugar, conviene tener muy claro cuál va a ser el objetivo de la internacionalización del territorio, de esta decisión depende la especialización que deberá alcanzar. No puede ser otro que posicionarse férreamente en la creciente globalización de la economía y la competencia internacional, en nuestro caso en el marco de la UE. Por ello se hace necesario recordar el mensaje central de la política de cohesión y sus instrumentos para el periodo comprendido entre 2007 y 2013, concretado en lograr mayor crecimiento y más puestos de trabajo en todas las regiones y pueblos de la Unión Europea.

3 MINISTERIO DE MEDIO AMBIENTE (marzo 2007): Libro Verde del Medio Ambiente urbano. Tomo I. Secretaría General Técnica. Centro de Publicaciones. Pp. 143. 
Llegados a este punto, y dado que todos los territorios con sus respectivas comarcas y regiones se marcan el mismo objetivo, surge la pregunta ¿con qué instrumentos diferenciadores puede alcanzar cualquiera de nuestros pueblos estos objetivos? La respuesta se encuentra en el contenido del proceso señalado, puesto que los patrones de la globalización son especialmente sensibles al atractivo físico del territorio, puede recurrir a la ventaja cualitativa de la historia del lugar, definida por su patrimonio material e inmaterial y que ha de conformar la nueva y competitiva imagen. Parodiamos de nuevo a AMENDOLA ${ }^{4}$ «Convertir la imagen del territorio en instrumento de un proyecto de desarrollo, descubrir el valor estratégico de la imagen territorial para posicionarse en la jerarquía emergente implica, en primer lugar, una profunda renovación de sus infraestructuras, en segundo lugar apostar por una especialización que posibilite la capacidad competitiva, por último, pero probablemente sea el paso más importante, es preciso concienciarse que el origen de todo proyecto debe ser de carácter endógeno, cuyos factores en equilibrio con los exógenos diseñarán las estrategias a seguir.

Esta complejísima labor conlleva una condición básica ineludible, por otra parte apuntada en el mensaje central de la política de cohesión de la Unión Europea en su «Panorama de los Reglamentos de 2007-2013 para las políticas regional y de cohesión». El nuevo modelo de desarrollo debe potenciar la capacidad de desarrollo tanto de la propia ciudad como de su ámbito territorial. Se impone abandonar la trasnochada idea de abordar únicamente la problemática de la capital regional o comarcal considerada en sí misma. El nuevo modelo de desarrollo rompe con la vieja dicotomía, propone como condición básica enmarcar a la capital regional o comarcal no solo en su conjunto urbano sino también en el ámbito territorial. Hay que reclamar cambios radicales en el gobierno del ámbito rural para incorporar esta condición básica en la complejidad de la ordenación espacial del territorio en el marco del nuevo modelo de desarrollo. Por esta razón, como veremos más adelante, una de las estrategias fundamentales para alcanzar los objetivos propuestos consiste en integrar el patrimonio en la vida cotidiana del municipio, levantar las barreras sociales que aíslan muchos núcleos históricos de la toma de decisiones sobre su futuro.

Por último, debe aludirse a los cimientos en los que se debe asentar el nuevo modelo de desarrollo que no pueden ser otros que la sostenibilidad. Como es sabido el concepto de desarrollo sostenible nace con los años setenta del pasado siglo y se afianza a través de diferentes informes, entre los que cabe destacar el del Club de Roma de 1972, el Informe Brandt de 1980, aunque es el de Brundtland de Naciones Unidas de 1987 el más conocido y aceptado, define el desarrollo sostenible como aquel modelo de desarrollo que satisface las necesidades presentes sin comprometer la capacidad de las generaciones futuras de satisfacer las suyas.

Para entender la trascendencia del nuevo modelo de desarrollo cimentado en la sostenibilidad resulta interesante recurrir a la Carta de Atenas en su punto 4 de la Parte A, La coherencia medioambiental: «Es una suerte vivir y trabajar en su propio ambiente, cerca de un patrimonio cultural y natural bien conservado (paisajes significativos, emplazamientos arqueológicos, monumentos, espacio rural y vecindades tradicionales, parques,

4 AMENDOLA, G, op.cit. pp. 292. 
plazas y otros espacios exteriores, lagos, ríos, litoral marítimo, reservas naturales) todo ello será cuidadosamente preservado y multiplicado. El urbanismo continuará siendo la medida más eficaz para conseguir proteger el patrimonio natural y cultural, y para producir las condiciones de creación de nuevos espacios libres que darán su coherencia a los tejidos urbanos».

\section{DISFUNCIONALIDADES DEL MEDIO RURAL}

La aprobación del primer Plan de Desarrollo Económico y Social en España, cuando acaban de arrancar los años sesenta de la pasada centuria, supone un cambio radical en la estructura de la producción y del empleo según preconizaba el Informe del Banco Mundial de 1961. El desarrollo se cimienta en la industrialización, lo que significa un brusco giro en la política del régimen esos momentos volcado a favor de los agricultores, cuyo patriotismo se contrapone al de los trabajadores industriales. Surgen los Polos de Promoción y Desarrollo Industrial, se distribuyen polígonos industriales por diversas ciudades, se potencia la industria difusa en pequeñas ciudades, de tal forma que junto a los centros hiperindustrializados, en que se convierten las grandes ciudades, las pequeñas se ven favorecidas por acciones puntuales de estos grandes centros, al mismo tiempo se ven afectadas por el brusco proceso desruralizador que surge como consecuencia de la crisis de la agricultura tradicional.

Se pone en marcha una dudosa planificación que estructura el territorio nacional en cuatro tipologías: áreas metropolitanas, ciudades de tipo medio, grandes zonas turísticas y áreas rurales. Tan solo tuvo aplicación lo referente a la primera tipología y se beneficiaron, como es lógico, Madrid, Barcelona, Valencia y Zaragoza, entre otras grandes capitales de provincia, aunque para el objetivo que se propone en este trabajo interesa resaltar estas cuatro. Teruel y su territorio quedaron en la más absoluta marginalidad a pesar de su estratégica posición. Adviértase que se hace referencia a los cuatro vértices del cuadrilátero noroeste peninsular en cuyo interior quedan enclavadas las tierras del Jiloca turolense. Con el apoyo político estos cuatro vértices actúan como centros de hiperconcentración industrial y urbana que atrae población de los espacios rurales encerrados en el cuadrilátero, convertidos ahora en centros de emigración porque siempre han sido centros sin planificación.

Las consecuencias de la dinámica demográfica que se pone en marcha son irreversibles y todavía presentes en el territorio. La provincia de Teruel en su conjunto queda estigmatizada como deprimida por la pérdida circunstancial de actividad, como consecuencia de una situación que se convierte en cíclica, las sucesivas políticas agrícolas e industriales significan serias recesiones. Todos los indicadores socioeconómicos vienen a confirmar que de la depresión se pasa al declive, resultado lógico del atraso estructural en el que permanece por ausencia de proyectos y planes de desarrollo integrado que posibiliten salir de la depresión. Un atraso estructural endémico que ha originado discursos, comisiones de estudio, publicaciones de especialistas y organismos creados por y para el desarrollo, pero el fenómeno parece haber hecho mella en los genes de cuantos todavía permanecen en el territorio y se dejan llevar por repetidos cantos de sirena. 
Un atraso estructural que la nueva gobernanza deberá atajar, entre otros, con las siguientes iniciativas:

- Puesta en valor de un espacio que se ha catalogado como desfavorable. Queda lejos el tiempo en el que se podía poner como escusa la altitud, la climatología, la angostura de los valles. Hay que considerar que las relaciones campo/ciudad han cambiado por las de ciudad/campo para generar un proyecto de futuro basado en la oferta de ocio. Es preciso imponer una nueva visión del paisaje rural, su puesta en valor debe equivaler a considerarlo como soporte de actividades económicas, sociales y medioambientales.

- Apostar por la multifuncionalidad agrícola. En el Preámbulo del Convenio Europeo del Paisaje (Florencia 20-X-2000) llama la atención un punto que es de sumo interés para el objeto de este trabajo, hace referencia a la multifuncionalidad agrícola y al punto anterior: «Tomando nota de que el paisaje desempeña un papel importante de interés general en los campos cultural, ecológico, medioambiental y social, y que constituye un recurso favorable para la actividad económica y que su protección, gestión y ordenación pueden contribuir a la creación de empleo».

- Urgente reciclaje en la formación: hoy la estructura de la población es regresiva y alcanza su máxima expresión en el envejecimiento de la población activa con su fuerte impacto en la productividad. Esta situación fuerza la necesidad de reciclaje en su formación. Además no puede ignorarse que esta estructura está marcada por la baja natalidad y el progreso de la longevidad. Los poderes públicos vienen preocupándose por las pensiones y los servicios de salud, pero no hacen nada por dar un sentido útil a las personas que integran la mal llamada tercera edad y muy poco para el reciclaje de la población activa, las escasas acciones llevadas a cabo no parecen suficientes, al menos no han dado el resultado apetecido.

- Aprovechar las nuevas infraestructuras para evitar el secular atraso: la dotación y características de las infraestructuras básicas, tanto intra como extraprovinciales, todavía adolecen de grandes deficiencias, lo que justifica la sensación de aislamiento, la postura más negativa para alcanzar un desarrollo armónico.

- Aprender de los procesos emigratorios y sus consecuencias: No puede olvidarse que el Valle del Jiloca ha soportado en el último medio siglo tres emigraciones brutales, la primera derivada de la modernización de la agricultura, la segunda impulsada por la crisis industrial que afecto a los «polos» industriales del territorio sin que se buscaran alternativas, la tercera está padeciéndola en estos momentos y tiene una relación directa con el acusado envejecimiento de la población.

- Atajar el deterioro social: emigración y envejecimiento son responsables directos del desfase técnico, social y cultural que padecen estos territorios.

Las disfuncionalidades señaladas plantean unas perspectivas muy complicadas para el mundo rural, porque siempre se ha mirado para otra parte, pero lamentablemente fueron puestas de relieve hace casi dos décadas por el Seminario «Situation et perspectives de l'Europe rurale»:

- Declive del empleo en el sector agrícola e importancia económica relativa de la producción alimentaria, 
- Mundialización de los elementos clave de la economía.

- Introducción y propagación de las nuevas tecnologías, particularmente de las tecnologías de la información y de las comunicaciones así como de la biotecnología,

- Los cambios demográficos desembocan en el envejecimiento de la población e incremento de las relaciones de dependencia,

- Movilidad personal creciente, a través de las migraciones cotidianas, migraciones definitivas, el turismo y las actividades de ocio,

- Emergencia de una nueva utilización del espacio rural, nuevas demandas sociales en cuanto al uso del suelo y del paisaje.

- Emergencia de nuevos ganadores y perdedores como consecuencia de los procesos de cambio.

- Emergencia del medioambiente como una potente fuerza política y ética.

- Fin de la guerra fría y emergencia de un nuevo orden mundial o ideología de «mercado libre».

- Búsqueda de nuevas formas de gestión que no se apoyan exclusivamente en el Estado sostenido por una élite burocrática, su busca mucho más la participación del conjunto de la población.

\section{EL PATRIMONIO COMO INSTRUMENTO DE DESARROLLO}

La Conventión pour la sauvegarde du patromoine architectural de l'Europe (Granada, 3,X.1985) en su artículo 1 señala. «Para los fines de la presente Convención, la expresión «patrimonio arquitectónico» es considerada comprendiendo los bienes inmuebles siguientes:

1. Los monumentos: todas las realizaciones particularmente destacables en razón de su interés histórico, arqueológico, artístico, científico, social o técnico, y comprende las instalaciones o los elementos decorativos que forman parte integrante de esas realizaciones;

2. Los conjuntos arquitectónicos: grupos homogéneos de construcciones urbanas o rurales destacables por su interés histórico, arqueológico, artístico, científico, social o técnico y suficientemente coherente para ser objeto de una delimitación topográfica;

3. Los paisajes: obras combinadas del hombre y la naturaleza, particularmente construidas y constituyen espacios suficientemente característicos y homogéneos para ser objeto de una delimitación topográfica, destacable por su interés histórico, arqueológico, artístico, científico, social o técnico.

Apostar por el patrimonio como instrumento de desarrollo exige reflexionar en torno a los dos interrogantes siguientes:

¿Quién tiene interés por el patrimonio?

¿Cómo valorizar el patrimonio?

El compromiso de responder a tan complejos interrogantes viene de las consideraciones institucionales acerca del Patrimonio que no van más allá del mero conservacionismo, 
sirvan de ejemplo las recomendaciones que hace la decimoséptima Conferencia General de la Organización de Naciones Unidas para la Educación, la Ciencia y la Cultura celebrada en París el año 1972, aceptadas por todos los países comprometidos en la conservación del patrimonio:

1. Constatando que el patrimonio cultural y el patrimonio natural están cada vez más amenazados de destrucción, no sólo por las causas tradicionales de deterioro sino también por la evolución de la vida social y económica que los agrava con fenómenos de alteración o destrucción aún más temibles.

2. Considerando que el deterioro o desaparición de un bien del patrimonio cultura y natural constituye un empobrecimiento nefasto del patrimonio de todos los pueblos del mundo.

3. Considerando que la protección de ese patrimonio a escala nacional es en muchos casos incompleto, dada la magnitud de los medios que requiere y la insuficiencia de los recursos económicos, científicos y técnicos del país en cuyo territorio se encuentra el bien que ha de ser protegido.

4. Teniendo en cuenta que la Constitución de la Unesco estipula que la organización ayudará a la conservación y la protección del patrimonio universal, y recomendando a los interesados las convenciones internacionales que sean necesarias para ese objeto.

5. Considerando que las convenciones, recomendaciones y resoluciones internacionales existen a favor de los bienes culturales y naturales, demuestran la importancia que tiene para todos los pueblos del mundo, la conservación de esos bienes únicos e irremplazables de cualquiera que sea el país a que pertenezcan.

6. Considerando que ciertos bienes del patrimonio cultural y natural prestan un interés excepcional que exige se conserven como elementos del patrimonio mundial de la humanidad entera.

7. Considerando que, ante la amplitud y gravedad de los nuevos peligros que les amenazan, incumbe a la colectividad internacional entera participar en la protección del patrimonio cultural y natural del valor universal excepcional prestando una asistencia colectiva que sin reemplazar la acción del Estado interesado la complete eficazmente.

8. Considerando que es indispensable adoptar para ello nuevas disposiciones convencionales que establezcan un sistema eficaz de protección colectiva del patrimonio cultural y natural de valor excepcional organizada de una manera permanente, y según métodos científicos y modernos.

\section{¿Quién tiene interés por el Patrimonio?}

Nuevamente resulta de interés parodiar a Amendola ${ }^{5}$ cuando señala que «Ha comenzado la época, aún vigente, de la reutilización y de la puesta en valor de viejos pueblos y pequeñas ciudades según principios diferentes de los del pasado: valorización de las

5 AMENDOLA, G., op. Cit. Pp. 31. 
diversidades, recuperación de los testimonios del pasado, búsqueda del genius loci y de las bases espaciales de la identidad de los habitantes, ligamen de lo bello con la funcionalidad».

En la reutilización y transformación participan tres figuras con diferentes intereses y hasta en ciertos momentos contrapuestos: quienes encuentran el placer de vivir en pueblos con fuerte sabor histórico, los turistas que buscan ocio y recreación entre la singularidad de una localidad histórica y, por último, la administración local y regional que aprovecha estos intereses para promover planes de dinamización de los núcleos históricos, con fines turísticos y de ubicación de actividades de la administración local, regional, educativa y de investigación.

Es evidente que desde hace algún tiempo se observa un fenómeno de incuestionable trascendencia para la recuperación y valoración de los pueblos. Un fenómeno que cabe definir por el gusto, la necesidad, el redescubrimiento de las señas de identidad del territorio, señas que están inscritas en el patrimonio histórico, en el más amplio sentido del término.

El centro histórico, depositario de la personalidad y el carácter de las gentes que actuaron en el escenario de su larga vida cotidiana, es buscado, casi podría afirmarse que anhelado, por una doble corriente de personas que, en definitiva, vienen a generar el mismo efecto, la misma necesidad, la misma demanda, la urgente recuperación de los pueblos con su rancio saber a un pasado más glorioso. La primera corriente está integrada por aquellas gentes que un día se dejaron seducir por la moda de vivir en los impersonales ensanches de las grandes ciudades, son personas que ahora redescubren, revaloran la vida tranquila del pueblo, la vida social más fácil y cercana frente a la neociudad de los barrios residenciales o cinturones industriales instalados en los espacios rurales que circundan la ciudad. Probablemente debería decirse que ante el fracaso de la periferia vuelve el gusto por el lugar acogedor y entrañable, aunque el nefasto proceso urbanizador ha dejado, en muchos casos, estos espacios a su suerte, todavía hoy encierran los testigos de un pasado esplendoroso.

La segunda corriente es la que pone en circulación la sociedad del ocio y la cultura en la que nos encontramos inmersos, en muchos de los mitos turísticos de los núcleos históricos da la impresión de que se acaba de descubrir que en ellos hay algo más que abandono, emigración, declive, envejecimiento, desfases sociales y culturales, aislamiento. Averiguan que sus pueblos, por muy sencilla que sea su historia, esconden los hitos y los nodos que permiten comprender, no sólo la realidad social que los ha producido, sino las raíces de una personalidad que durante demasiado tiempo está corriendo el riesgo de ser anulada. Esta corriente tiene la particularidad de generar una necesidad a esta nueva sociedad, una necesidad que demanda consumo y el patrimonio se convierte en un producto que cada vez interesa más a mayor número de personas, que su «consumo» genera una transversalidad muy rentable.

En esta doble corriente se produce una curiosa paradoja: ambas se necesitan para lograr sus fines y precisan de las actuaciones de la tercera figura, la administración que asumirá la responsabilidad de satisfacer a unos y a otros. Tanto unos como otros exigen un entorno de calidad como elemento indispensable para conseguir el éxito, la respuesta a tales exigencias sólo puede venir de las estrategias de la administración, de saber aunar sinergias a favor de recuperar la función de centralidad que un día no muy lejano ejercieron algunos núcleos rurales hoy sumidos en una profunda depresión. Tarea que requiere fuertes operaciones de rehabilitación y revitalización que tan sólo son posibles 
en el marco de una planificación que surja del seno de las diferentes administraciones implicadas. Sin embargo, la práctica totalidad de las fórmulas aplicadas se apoyan en la legislación sobre el suelo, una legislación que carece de imaginación y va muy por detrás de las necesidades, utiliza figuras que habitualmente tropiezan una y otra vez con una realidad que las supera ampliamente lastradas por la pesada burocracia que deja obsoletas muchas de las necesarias iniciativas.

\section{¿Cómo valorizar el patrimonio?}

El capital más importante de nuestros pueblos está constituido por todo cuanto en ellos es permanente, profundo y completamente enraizado en sí mismos. Disponer de este capital exige establecer un delicado equilibrio entre tradición y modernidad, entre el saber hacer local y las demandas de un desarrollo sostenible. Un equilibrio que sepa renovarlos al propio tiempo que hacerlos atractivos, pero con la condición de servir tanto a los visitantes como a los propios residentes en el pueblo. Cuando únicamente se piensa en convertir el capital-imagen en un mero producto de consumo, se está produciendo un fraude a la sociedad y una traición a la cultura popular que desemboca en el ocaso físico del pueblo. Por todo ello, la respuesta a este interrogante se nos presenta más compleja y es preciso buscar la respuesta a través de una reflexión en torno a dos elementos de actuación, en primer lugar cuanto significa innovar el capital-imagen de la localidad y, en segundo lugar, la trascendencia que tiene la calidad en todo intento de valorización de este capital-imagen.

El punto de partida debe ser la afirmación de que el capital-imagen de nuestro pueblo está constituido por el paisaje que se percibe al recorrerlo y sus elementos fisonómicos y estructuradores. Un paisaje interpretado bajo la idea expresada por LEFEBVRE ${ }^{6}$, según la cual el paisaje debe ser utilizado como el texto que permita comprender el contexto en el que se configura la realidad social que lo ha producido. Un texto que hará comprender el pasado, la personalidad de lo local, y más concretamente el saber hacer de ayer y de hoy. Tarea ignorada por muchos responsables municipales que tan sólo piensan en sus propios intereses, en la mayoría de las veces son de un oportunismo político desdeñable, se limitan a la rehabilitación interesada cuando no indiscriminada de algún elemento del patrimonio material «mayor», dejando en el olvido el que cabría denominar como «menor» y a la importantísima riqueza que constituye el inmaterial.

La descripción geográfica racional de la fisonomía de todos nuestros pueblos permite revelar una curiosa originalidad, derivada de la dinámica urbana sostenida a lo largo de diferentes etapas por las que ha transitado la configuración de su plano actual. La originalidad de cada localidad se concreta en la yuxtaposición de tramas que definen los diferentes barrios que lo integran. Yuxtaposición que es responsable de la acusada pérdida de legibilidad pero también de la falta de articulación entre los barrios medievales y los ensanches de los siglos XVIII al XX, fenómeno que, en no pocas ocasiones, origina serios problemas sociales, económicos y hasta de movilidad.

6 LEFEBVRE, H. (1968): Le droit à la ville. Anthropos. Paris. 
Los hitos de la historia constituyen hechos que confieren originalidad y distinción al plano del núcleo primitivo, de ahí su valor como capital y la necesidad de hacer todos los esfuerzos precisos para conservar el núcleo original. Sin embargo, no puede ignorarse que esta personalidad, que la originalidad percibida al transitar por sus calles, se ve impregnada por la reiterada interacción de cuatro categorías de factores -económicos, sociales, culturales y políticos- mudantes en el tiempo y en el espacio, pero siempre permanentes, con sus momentos de esplendor y sus profundas inflexiones, que dejan su impronta en el paisaje. Una impronta positiva/negativa que exige aprovechar la primera y rectificar la segunda, si ello es posible, o cuando menos hacer todos los esfuerzos para minorar los impactos indeseados.

En la originalidad histórica de todos nuestros pueblos se descubre una curiosa coincidencia, en todos ellos se distinguen dos claras etapas definitorias de su actual morfología, la primera en la que predomina el proceso de configuración del núcleo central y la presente caracterizada por la expansión del primitivo núcleo mediante barrios nuevos, en algunos casos con las características propias de los barrios industriales que surgen en las inmediaciones de la industria.

Los impactos de la etapa actual son palpables cuando se recorre cualquier núcleo histórico. La atonía urbanística con la que se llega a los años setenta se rompe bruscamente en el decenio siguiente como consecuencia de la aparición del gusto por los barrios construidos mediante bloques monocordes y viviendas adosadas surgidas de una encorsetada planificación urbana, fruto de una legislación creadora de figuras y modelos que en estos espacios tenían poca o ninguna cabida. Sin embargo, el academicismo y el clientelismo político y cultural de los redactores de los distintos instrumentos de planificación han dado lugar a efectos contradictorios y en, cierta medida, muy negativos. Han potenciado el deterioro del centro primitivo por un doble motivo, primero, por incapacidad para rehabilitarlo y revitalizarlo, y, segundo, por ser un espacio, en muchos casos, poco generador de plus-valías. Además, esta política urbanística impulsa una desmesurada expansión de los pueblos, se advierte en el plano heterogéneo que constituye la trama urbana moderna de cualquiera de ellos, expansión que viene a albergar a todos cuantos abandonan el cada vez más deprimido centro antiguo.

La complejidad de la legislación urbanística y los intereses de los gremios planificadores, unidos a las angustiosas prisas porque todos los municipios tengan alguno de los instrumentos de planeamiento recogido en la legislación estatal, han llenado el panorama urbanístico de normas, disposiciones y directrices, difíciles de desarrollar en unos espacios que han soportado un cúmulo de errores. Este continuo hacer y deshacer ha conducido a una cierta desorganización de la vida cotidiana, presa del urbanismo operativo más recalcitrante, hoy completado por el deseo de residir en contacto con la naturaleza. A pesar de tanta legislación, de complejas figuras, planes de reforma interior y de movilidad, de la premura para abordar problemas y buscar soluciones, se ha llegado a una curiosa paradoja, el núcleo originario aparece como encorsetado para efectuar las acciones positivas para el desarrollo, al mismo tiempo se extiende mediante una política edificatoria a base de barriadas de bloques construidos de forma industrial mediante la autoconstrucción. 
Conviene insistir que la propuesta del paisaje, con todos sus elementos, como instrumento de desarrollo no es un intento de querer verlo como un museo natural, frío y despersonalizado. La consideración de capital-imagen exige percibirlo como un organismo vivo capaz de cursar esa historia vivida en un largo recorrido de siglos. Es un espacio cambiante que evoluciona hacia una nueva actividad, al que ahora se le propone la funcionalidad bien de atractivo turístico, residencial, educativo o investigador, para devolverle un papel dinamizador de la vida rural en su conjunto, que implica una imaginación de planteamientos en busca de una planificación para que las calles recuperen su rancio sabor y su pasada vitalidad. Esta devolución puede lograrse con la propuesta de convertirlo en el más importante recurso económico del medio rural.

A la revitalización y revalorización del capital-imagen del medio rural puede llegarse por tres caminos no excluyentes sino complementarios y concurrentes. En primer lugar, se impone renovar las estructuras, modernizarse para afrontar la problemática, en este sentido convertirse en destino turístico, incluso en no pocas ocasiones puede ser de peregrinaje/turístico, es una buena solución aunque no la definitiva. En segundo lugar, es preciso buscar una especialización que lo haga fuerte y competitivo, la recuperación de un edificio singular con fines religioso-turístico, educativo o de ocio, piedra angular en la configuración de la nueva imagen, puede dotar al pueblo de instrumentos suficientes para la especialización dentro de una diversificación, como corresponde a la transversalidad que impregna el turismo. Por último, y este es el paso más importante, debe partir de la idea de que el desarrollo territorial endógeno es la más acertada de las soluciones posibles para la evolución sostenible del ámbito rural.

Tomada la decisión de modernizarse a través de las opciones apuntadas, es preciso tener muy en cuenta la regla de oro de toda planificación, cualquiera que sean sus fines: un entorno de calidad constituye el elemento indispensable para transformar un espacio en destino turístico-religioso, de ocio. Son abundantes las publicaciones y manifestaciones de todo tipo que ponen de relieve los factores determinantes en la elección del producto a consumir. En todas ellas se constata que un entorno de calidad y no contaminado constituye la primera razón de ser de todo intento por valorizar el núcleo histórico. Pero también se advierte que este entorno perecedero, difícil de reconstruir y ofertado sin ninguna medida puede ser aniquilado por la especialización que se adquiera a través de la nueva funcionalidad. Una interesante línea de actuación que propone el Libro Verde del Medio Ambiente Urbano consiste en insertar en los procesos de rehabilitación de los centros históricos y barrios tradicionales, actuaciones de recuperación de espacios verdes, ya sea en sustitución de edificios industriales o de viviendas que han perdido su función o que han quedado obsoletos.

Estas reflexiones ponen de relieve que la principal acción de todos los actores y sus estrategias, en la valorización del capital-imagen de los pueblos, debe encaminarse hacia la recuperación de un entorno dotado de una calidad indiscutible. Pero crear un entorno de calidad no significar colocar unos bancos, algunos maceteros y papeleras en ciertos nodos e hitos y dejar que amplias plazas se conviertan en aparcamientos y que la superficie se «privatice» a base de terrazas de bares y cafeterías con sus negativos impactos ambientales que llevan consigo la anulación de la tradicional función social que siempre se le atribuye a la plaza, además, los ruidos y otras molestias colaterales 
acaban por expulsar a la función residencial. La recuperación del entorno adecuado a las exigencias de la nueva funcionalidad debe pasar, como mínimo, por las siguientes acciones:

Preservación del legado arquitectónico, en el que se incluye el trazado de las calles, por cuanto constituye la razón de ser de la imagen de la localidad. Un legado arquitectónico que ha soportado profundos ataques del abandono de numerosos edificios, pero particularmente de las ideas defendidas por el urbanismo operativo. Un urbanismo que se caracteriza por las profundas transformaciones que introduce en la fisonomía de todos nuestros pueblos. Se suceden planes y normas generales casi siempre contradictorias, que auspiciados por mejorar las condiciones de habitabilidad van a mostrar el nulo respeto que se ha tenido por el patrimonio en su conjunto. Este urbanismo operativo, junto al descontrolado crecimiento, genera un plano que nada tiene que ver con la tradicional construcción, emprende arriesgadas operaciones de modificación, remodelación del espacio interior de los viejos cascos históricos, ocasionando mutilaciones irreparables, tanto desde el punto de vista de la fisonomía como de su funcionalidad. Una corriente devastadora se abrió paso afirmando que la trama urbana heredada del pasado medieval era un serio inconveniente para el desarrollo y la modernidad. La consecuencia fue el abandono y la destrucción de no pocos cascos históricos y la aparición de un nuevo plano que viene a sustituir al viejo trazado.

Una planificación integrada del conjunto urbano que abarque, como mínimo, los siguientes aspectos:

Rehabilitación: como fórmula de intervención capaz de mantener y recuperar todos los elementos que configuran la imagen de la localidad, entendida aquí como el conjunto de elementos arquitectónicos pero también por la propia historia que lo envuelve.

La intraurbanización capaz de dotar al centro histórico de una funcionalidad que genere la gentrificación ansiada. La operación más acertada de intraurbanización se concreta en dotar al centro del suficiente atractivo, además de plantear estrategias que den prioridad a las necesidades de los peatones y los habitantes y no a los conductores que tan sólo pretenden atravesar la zona. Esta fuera de toda duda que si se logra devolver al centro su pasado funcional completado con la nueva funcionalidad, su vitalidad a través de un complejo combinado intraurbanización/gentrificación, habrá merecido la pena pensar en este recurso como instrumento para el desarrollo.

Resulta paradójico comprobar como la expansión de muchos de nuestros pueblos es totalmente contradictoria con su propia historia, atenta claramente a los intereses que configuran su mito. Urge abandonar la nefasta planificación que induce a huir de los centros trasladando a la población a una periferia donde el automóvil se convierte en pieza indispensable.

En los núcleos con largo pasado histórico es lógico el desplome de edificios que dejan en el paisaje urbano abundantes eriales sociales. Unos solares que vienen soportando una débil demanda pero que ante las operaciones de intraurbanización aparece la idea de obtener interesantes plus-valías. Entre tanto se convierten es espacios desdeñables cuyo abandono contribuye a desdibujar la imagen patrimonial del pueblo. 
Resulta del todo punto imprescindible potenciar un diseño armónico si se quiere «vender» la localidad. La calidad visual de muchos de estos espacios es bastante deprimente. La generalización de la uralita causa daños a una arquitectura portadora de profundas particularidades. Es muy frecuente descubrir autoconstrucciones o autorreconstrucciones sin ninguna garantía de calidad.

Atajar la contaminación urbana. Con más frecuencia que la deseada muchos de nuestros pueblos adolecen de un esmerado cuidado de sus calles más antiguas, tales como recogida de basura, eliminación de residuos, etc., a los que contribuye la existencia de los citados eriales sociales. Por otra parte, la baja densidad de habitantes ha dado lugar a que las viejas casas mejor conservadas se conviertan en locales de ocio poco recomendables, donde el ruido se manifiesta como elemento distorsionador.

En conclusión, un mundo rural que tanto espera del nuevo modelo de desarrollo, está obligado a buscar una planificación integrada del territorio y su función con un doble objetivo, de una parte evitar los impactos negativos y, de otra, aprovechar las potencialidades como instrumentos capaces de renovar y poner en valor el paisaje, no sólo restaurando su patrimonio sino revitalizando sus espacios más singulares. Conviene hacer hincapié en que los medios especializados no dudan en vaticinar a este modelo de desarrollo una espectacular emergencia. Por esta razón la mayoría de los pueblos tienen ante sí un futuro con el que no habían contado, pero es preciso abordar operaciones complejas para que resulten más atractivos.

\section{ACTORES Y ESTRATEGIAS PARA LA NUEVA FUNCIONALIDAD}

Autorizadas voces advierten de las serias dificultades con que se tropieza a la hora de dominar el futuro del medio rural. Dificultades que se incrementan en la problemática que venimos planteando en este trabajo, pues se trata de transformar antiguos equipamientos en estructuras modernas para ejercer un doble papel, por una parte deben convertirse en elementos clave de la nueva estructuración de las prácticas sociales y, por otra, desempeñar un cometido decisivo en la valoración del patrimonio a través de un complejo proceso de rehabilitación, recuperación y revitalización. En este propósito se ven comprometidos tanto los urbanistas, como los empresarios y las instituciones y colectivos locales que afrontan las exigencias de un nuevo modelo de desarrollo sobre el que pretenden consolidar el porvenir del territorio en su conjunto. El artículo 14 de la citada Convención señala «En vista de secundar la acción de los poderes públicos a favor del conocimiento, protección, restauración, mantenimiento, gestión y animación del patrimonio, cada parte se compromete:

1. A poner en marcha, en los diversos estados de los procesos de decisión, estructuras de información, de consulta y de colaboración entre el Estado, las colectividades locales, las instituciones y asociaciones culturales y el público;

2. A favorecer el desarrollo del mecenazgo y de las asociaciones con fines no lucrativos.

En esta situación la cuestión estriba en marcar las estrategias capaces de aunar todas las sinergias locales en la recuperación patrimonial y funcional del pueblo. Tarea que 
conlleva un conjunto de soluciones prácticas a problemas concretos que cabe sintetizar en el deseo de que los pueblos dejen de ser utilizados únicamente para trabajar y divertirse. Objetivo tal vez demasiado ambicioso porque requiere armonizar economía, calidad ambiental y bienestar social en un espacio que, además de ser depositario del Patrimonio ejerza como espacio moderno en base a una nueva y exigente funcionalidad. Un ambicioso objetivo que, a nuestro juicio, exige poner en práctica la estrategia siguiente: Integrar el patrimonio en el conjunto territorial:

La estructura y articulación de los espacios rurales deben mucho al fuerte impacto de la historia que les ha tocado vivir. Entre unos momentos y otros, distantes y distintos, la sucesión de factores, con su complejo juego, se encargan de modificar el escenario de la vida cotidiana; los tramoyistas adaptan el entramado a las nuevas necesidades, a las exigencias de la nueva representación funcional, pero no pueden evitar dejar profundas huellas, en no pocas ocasiones indeseadas, que configuran el paisaje que visitantes y nuevos residentes encuentran. Derribo de castillos y murallas, ensanches, anexiones de antiguos arrabales, remodelaciones de casas solariegas, instalaciones religiosas, militares e industriales, procesos bruscos de desvinculación de éstas funciones, interesada terciarización de la vida cotidiana, vienen a dibujar un territorio heterogéneo que con el paso del tiempo exige fuertes inversiones económicas y origina elevados costos sociales y medioambientales.

Son numerosas los pueblos que al recorrer sus calles revelan como su dilatada historia ha sabido reunir y asentar en su solar a un conjunto de actividades y funciones que han modelado y remodelado continuamente el paisaje. Esta sucesión de factores dejan en la práctica totalidad de los pueblos áreas claramente diferenciadas pero desestructuradas entre sí, presentan circunstancias muy particulares que exigen ser abordadas con un planeamiento que sea al mismo tiempo particularizado e integrador en su conjunto. Sin embargo, se están elaborando tantos planes parciales como áreas singulares se detectan, lo que imposibilita un tratamiento de conjunto que integre todo el patrimonio en la vida cotidiana.

Una panorámica general permite clasificar estas áreas en las siguientes tipologías:

- áreas vacías: son aquellas en las que prácticamente ya no quedan edificaciones por los sucesivos derribos que los ayuntamientos han ido ejecutando para desplazar la marginalidad física y social. Cuando todavía subsisten inmuebles se descubre un abandono total, carecen de interés económico y presentan una débil densidad. Generalmente estas áreas ocupan el centro originario del pueblo y en su suelo esconden buena parte del patrimonio de un pasado glorioso. La intervención en estas áreas despierta interesantes y polémicos debates que no contribuyen a soluciones eficaces por la radicalización de posturas.

- áreas malformadas: se definen por el largo proceso de deterioro que presenta su morfología, circunstancia que ha originada la salida de las categorías sociales con alguna posibilidad económica así como la práctica desaparición del comercio tradicional. Registran una baja densidad demográfica que año tras año sigue perdiendo potencial. Es palpable el continuo envejecimiento de los inmuebles 
y de la población residente, los servicios muestran un deprimente déficit. Demandan urgente intervención que debe ir más allá de la mera rehabilitación, exigen conservar las características principales del tejido existente así como de la arquitectura.

- Áreas obsoletas: ocupan amplias superficies dispersas y surgen como consecuencia de la pérdida de funcionalidad -instalaciones militares, conventos e iglesias, colegios, estaciones de ferrocarril, antiguas fábricas, instalaciones mineras, etc.han dejado de prestar sus servicios, en el caso de que todavía alguno desarrolle cierta actividad resulta factible desplazarla a otro espacio más adecuado. La ubicación de estos inmuebles y la superficie que ocupan los convierten en objetivos interesantes para integrarlos en un desarrollo en el que pasen a jugar un nuevo papel.

En esta situación, parece meridianamente claro que todos los actores de la vida cotidiana de la localidad deben tener una preocupación común: la recuperación del conjunto urbano mediante una emergente funcionalidad. Para ello es preciso recurrir a una planificación integrada que se proponga como base para actuar un doble compromiso, los edificios singulares representativos de funciones hoy obsoletas deben ser abordados con las ideas que propugna la intraurbanización, convertirlos en instrumentos básicos de la nueva funcionalidad para que irradien en su entorno la figura de la gentrificación, que consiste en la atracción de población de grupos sociales que pueden integrarse perfectamente en la nueva estructura.

Un planeamiento que conecte con la filosofía que se plantea en este trabajo debe seguir una serie de pasos. El primer paso es definir el Plan como un documento integrador de todas las políticas con incidencia en el pueblo es una referencia clara a los mensajes clave expuestos en el apartado anterior. Para alcanzar este objetivo es preciso profundizar en el conocimiento de los problemas existentes que configuran el territorio actual y las tensiones que se producen en su territorio. Basados en los problemas detectados puede proponerse un modelo territorial y urbano equilibrado, con actuaciones diseñadas a largo y corto plazo, con el objeto de solucionar los problemas fundamentales de funcionamiento que presentan, así como las interrelaciones medioambientales no deseadas que se producen en el territorio.

El nuevo modelo territorial se definirá en base a una serie de criterios y objetivos muy concretos. Los criterios se apoyan en tres aspectos básicos:

- La protección medioambiental es uno de los pilares básicos de la política territorial, que tiene, entre otros, los objetivos de hacer compatibles y complementarios el desarrollo y la conservación de los recursos.

- Se tendrá esencialmente en cuenta la aplicación de la legislación y normativa vigente en materia de protección y conservación medioambiental (normativa europea, nacional y regional).

- La necesidad y la especial utilidad de la realización de Estudios de Impacto Ambiental para aquellas actividades de nueva implantación cuyo desarrollo debe realizarse siempre con las premisas de respeto y conservación del medioambiente y el patrimonio. 
Los criterios apuntados dan lugar a la formulación de media docena de objetivos fundamentales que, de nuevo, concuerdan claramente con cuanto se viene afirmando en nuestro trabajo: Compativilizar el desarrollo del municipio con la conservación de los recursos; valorar la presencia de especies, hábitats, endemismos, árboles singulares, etc., en cada una de las áreas territoriales de las que se compone el término municipal, con el fin de fijar las protecciones correspondientes; delimitar áreas homogéneas que reúnan valores naturales y medioambientales similares, estableciendo medidas de protección adecuadas al objeto de protección; identificación y delimitación en lo posible de las áreas arqueológicas de interés, con el objeto de fomentar su protección y salvaguarda; catalogación de los elementos de interés patrimonial y cultural, con el establecimiento de medidas tendentes a su conservación y/o recuperación.

Una vez que se pone de manifiesto el interés por considerar al conjunto municipal como un todo para definir la calidad de vida, se pueden proponer unos objetivos de amplio alcance que cubren los grandes retos a los que el medio rural tiene que enfrentarse. Estos objetivos se concretan del modo siguientes: resolución prioritaria de los problemas detectados; regeneración de los barrios consolidados, estableciendo medidas que compaginen los procesos de conservación y cambio; protegiendo la estructura de la trama urbana edificada de las áreas de mayor interés arquitectónico y facilitando en otros casos la renovación; potenciar la integración de los distintos barrios que lo componen, de forma que se configure un conjunto al que los barrios aporten su diversidad intrínseca; tratamiento pormenorizado de las barreras físicas que separan los barrios entre sí (río, ramblas, ferrocarril, carreteras), instrumentando medidas y actuaciones concretas que minimicen los impactos negativos; asunción de los preceptos establecidos en la figura denominada Plan Especial de Protección, engarzando estos con el tratamiento de las áreas colindantes al ámbito del Plan Especial con el fin de aunar criterios similares evitando los puntos de fricción; conservación y respeto, con carácter general, de las alineaciones existentes, completando las que sean necesarias para la mejor definición de las áreas sin consolidar, y modificando las que fueran precisas para incorporar las nuevas ordenaciones que se proponen; distribución homogénea de los equipamientos en el conjunto del territorio, equilibrando la localización de dotaciones y servicios en las distintas zonas, teniendo en cuenta el carácter particular de cada barrio, de forma que se atienda a sus demandas específicas; tratamiento pormenorizado de los conjuntos con identidad propia, propiciando el mantenimiento de sus tipologías; establecimiento de una distribución de usos y tipologías coherente, evitando la consolidación de pequeñas zonas aisladas dentro de la estructura del pueblo, asimilando los saltos tipológicos existentes con propuestas de integración hacia el tejido consolidado o pendiente de transformación; completar la red de espacios libres y zonas verdes con nuevas áreas que permitan conseguir, junto con los parques y jardines existentes, un sistema de espacios libres integrado en la trama callejera que contribuya a relacionar las distintas partes del territorio entre sí; incorporación al tejido de las áreas consolidadas parcialmente por la edificación que se sitúan colindantes, dotándolas de una ordenación pormenorizada que se integre plenamente con el conjunto; previsión de nuevas áreas de crecimiento, seleccionando aquellas zonas que contribuyan a mejorar la estructura global que sustenta el entramado de sus calles, absorbiendo en su caso enclaves existentes; conservación, fomento y establecimiento 
de medidas específicas para la rehabilitación del patrimonio histórico, artístico, cultural y arqueológico; fomentar la oferta de suelo para promociones de viviendas sujetas a algún régimen de protección.

\section{CONCLUSIONES}

Los pueblos y pequeñas ciudades históricas del medio rural español deseosas de ocupar un puesto decisorio en la creciente competencia internacional, que les permita sacudirse la pesada losa que el desarrollismo y su aliado el urbanismo operativo han dejado caer sobre ellas, tienen la obligación, en el marco de un modelo de desarrollo sostenible, de asumir con todas sus consecuencias los siguientes mensajes:

* El posicionamiento en la jerarquía territorial emergente, tanto a nivel internacional como nacional y regional, no puede ser a cualquier precio, sólo es posible si se busca a través de un desarrollo sostenible capaz de afrontar impactos indeseados que corroerán los cimientos de su historia. Pero con una salvedad inviolable: la búsqueda de un desarrollo sostenible no es solamente la firma de conciertos de sostenibilidad y luego permitir construcciones indiscriminadas que socavan la fragilidad de los núcleos originarios del pueblo. Un posicionamiento de esta naturaleza exige conocer su capitalimagen, esto es, todo cuanto de permanente, profundo y entrañable hay en el pueblo. Un capital-imagen que plantea demandas de calidad indiscutibles. No es suficiente encontrar frases bonitas y llamativas desde el punto de vista comercial pero que luego chocan con la realidad, no aportan nada a la revitalización del patrimonio local.

* Enfrentarse a las actuales disfuncionalidades no es tarea fácil y mucho menos como vienen actuando muchas de las autoridades responsables del patrimonio pero también de la vida en los pueblos, se limitan a recuperar, a poner en valor un elemento del llamado patrimonio mayor y olvidan por completo todo cuanto comporta el patrimonio menor, el patrimonio inmaterial. Así puede comprobarse como muchos pueblos se fragmentan, una parte aparece embellecida y el resto continúa en su malformación y obsolescencia, * Sin la movilización de las sinergias locales es francamente imposible alcanzar la integración de las preocupaciones medioambientales, sociales, económicas y culturales. Unas sinergias locales que se fundamentan en su saber hacer local para procurar una localidad que satisfaga tanto a los visitantes como a los residentes. Es preciso romper con la inercia mantenida hasta hoy por un buen número de pueblos que esconden una pesada carga histórica. Inercia que se caracteriza por limosnear apoyos económicos de las administraciones de ámbito territorial superior para acciones puntuales. Acciones todas ellas que deben estar en la mente de aquellos responsables. A la ruptura con esta disfuncionalidad se puede llegar con éxito a través de crear una imagen nueva del pueblo que impulse y desarrolle una actividad capaz de convertirse en el anhelado desarrollo. El pueblo rural es, sin duda, uno de los sistemas más complejos creados por el hombre por su diversidad y tipología del emplazamiento. Hoy, además, los procesos de distinta naturaleza, económicos, sociales, culturales, tecnológicos, funcionales, etc., hacen que esa complejidad se haya visto acentuada, provocando conflictos y disfunciones que son cada vez más insolubles con el uso 
de lógicas lineales y visiones parciales de la realidad. Por otra parte, la naturaleza global de parte de los problemas relacionados con la sostenibilidad, o la economía, o la entrada en la nueva era del conocimiento, obligan a extender la complejidad de las soluciones a escalas mayores, trascendiendo las escalas netamente locales o regionales. ${ }^{7}$

\section{BIBLIOGRAFÍA}

ABDEL MOUR, A. (1982): Introduction a l'histoire urbaine de le Syrie otomane. Université Libanaise. Beyrout.

ABU-LUGHOD, J. (1976): Development in North African urbanism: the process of decolonisation. Northwestern University.

AÏSSANI, D. (1999): Rôle de la Société civile dans l'Aménagement urbain: cas de la ville de Béjaïa. Actes du Cours - Séminaire «Urbs et Civitas». Alghero-Sardaigne.

ALGRIN, T. (2000): «Lemonument est-til soluble dans l'urbain?». In Villes d'hier, villes d'aujourd'hui en Europe. Fayard. Paris.

AMENDOLA, G. (2002): La ciudad postmoderna. Celeste Ediciones. Madrid.

ANDRÉS SARASA, J. L. (2000): Cambio de estructuras en un municipio agrourbano; Santa Eulalia del Campo .Centro de Estudios del Jiloca.Zaragoza

ANDRÉS SARASA, J. L. (2004): «Innover l'image de la ville historique comme stratégie pour le développement soutenable. Lorca atelier du temps». VII Seminaire International. Conference Permanente des villes Historiques de la Mediterranée. Lorca.

ASCHER, F. (1995): Métapolis ou l'avenir des villes. Odile Jacob. Paris.

BAILLY, A. S. (1979): La percepción del espacio urbano. Col. Nuevo Urbanismo. Instituto de Estudios de Administración Local. Madrid.

BASTIE, J. et DEZERT, B. (1991): La ville. Masson. Paris.

BEAUCHARD, J. (2000): La Bataille du territoire: mutation spatiale et aménagement du territoire. L' Harmattan. Paris.

BENÉVOLO, L. (1993). La Ville dans l'histoire européenne. Seuil. Paris.

BOUINOY, J., BERMILS, B. (1995): La gestion stratégique des villes. Entre compétition \& coopération. Armand Colin. París.

BRUN, J, RHEIN, C. (1994): La Ségregation dans la ville. L'Harmattan. París.

CALVINO, I. (2002): Las ciudades invisibles. Siruela. Biblioteca Calvino. Madrid.

CHALINE, C. (1989): Urbanisme et developpement. SEDES. Paris.

CHALINE, C., DUBOIS MAURY, J. (1994): La Ville et ses dangers - Prévention et gestion des risques natureles, sociaux et technologiques. Masson. Paris.

CONFERENCE PERMANENTE DES VILLES HISTORIQUES DE LA MEDITERRANEE (2004): Acteurs locaux et patrimoine immaterial: le rôle des Villes historiques de la Méditerrané. ISPROM-PUBLISUD. Paris.

COURSON, B. (1003): Le Projet de ville - Essai pratique. Syros. París.

7 MINISTERIO DE MEDIO AMBIENTE. (2007): Libro Verde del Medio Ambiente Urbano. Tomo I. Red de Redes de Desarrollo Local Sostenible. Pp.172. 
DUBET, F. Et. All. (1994): Universités et villes. L'Harmattan. Colecction villes et entreprises. Paris.

FRIEDMANN, J. y WEAVER, C. (1981): Territorio y función. Col. Nuevo Urbanismo. Instituto de Estudios de Administración Local. Madrid.

GARCÍA MARCHANTE, J. S. y TROITIÑO VINUESA, M. A. (coord) (1998): Vivir las ciudades históricas. Recuperación integrada y dinámica funcional. Colección Estudios. Ediciones de la Universidad de Castilla-La Mancha. Cuenca.

HALL, P. (1996): Ciudades del mañana. Ediciones del Serval. Barcelona.

LEVY, J. P. (1987): Centres villes en mutation. C.N.R.S. Paris.

MAICHE, B. (2003): Décision politique et mutation urbaine, le cas de Mila. Thèse de Magíster en urbanisme. Constantine.

MARC, W. (1999): La Transition urbaine ou le pasaje de la ville pedestre à la ville motorisée. Mardaga. Liège.

MOLINA MOLINA, A. L. y ANDRÉS SARASA, J. L. (2007): La Ciudad Histórica (cuatro ejemplos murcianos). Real Academia Alfonso X El Sabio. Murcia.

O.C.D.E. (1994): Des villes pour le 21e siécle. Paris.

PAQUOT, T.; LUSSAULT, M.; BODY-GENDROT, S. (2000): La ville et l'urbain. I'état des savoires. Editions la Découverte. Paris.

POLÉSE, M. et SHEARMUR, R. (2005): Economie urbaine et regionjale. ECONOMICA. Paris.

PIERRE, V. (1996): Mondialisation, villes et territoires. PUF. Paris.

PROST, A. (1992): Éducation, societé et politiques. Seuil. Paris.

PUMAIN, D. (1982): La dinamique des villes. ECONOMICA. Paris.

III SEMINARIO INTERNACIONAL DE LA CONFERENCIA PERMANENTE DE CIUDADES HISTÓRICAS DEL MEDITERRÁNEO (1999): La ciudad elemento de identidad y factor de desarrollo del Mediterráneo: historia y perspectivas. Excm. Ajuntament de Xätiva. Valencia.

SOEUR, J. P. (1999): Changer la ville. Pour une nouvelle urbanité. Odile Jacob. Paris. TRIBILLON, J. F. (1991): L’Urbanisme. La Decouvert. París. 